\title{
Multiple electrode aggregometry in thrombocytopenic haemato-oncologic patients - Influence of haematologic variables
}

\author{
Elin Netland Opheim ${ }^{1,2}$, Torunn Oveland Apelseth ${ }^{2,3}$, Simon J. Stanworth ${ }^{4}$, Geir Egil Eide ${ }^{5,6}$ and Tor Hervig ${ }^{1,2 *}$ \\ ${ }^{1}$ Department of Clinical Science, University of Bergen, Norway \\ ${ }^{2}$ Department of Immunology and Transfusion Medicine, Haukeland University Hospital, Bergen, Norway \\ ${ }^{3}$ Laboratory of Clinical Biochemistry, Haukeland University Hospital, Bergen, Norway \\ ${ }^{4}$ NHS Blood and Transplant/Oxford University Hospitals NHS Trust, John Radcliffe Hospital, Oxford, UK \\ ${ }^{5}$ Department of Global Public Health and Primary Care, University of Bergen, Norway \\ ${ }^{6}$ Centre for Clinical Research, Haukeland University Hospital, Bergen, Norway
}

\begin{abstract}
Introduction: Clinical experience with Multiplate aggregometry is limited in thrombocytopenic, haemato-oncology patients.

Objectives: The primary objective of this prospective observational pilot study was to characterise changes in Multiplate area under the curve related to variations in platelet count. Secondary objectives were to characterise changes in Multiplate area under the curve related to variations in the haematological variables white blood cell count, haemoglobin concentration, mean platelet volume, and reticulated platelet percent and count.

Materials: Ten thrombocytopenic, haemato-oncology patients were included. Haematological measurements were performed daily, and Multiplate analyses on weekdays.

Results: Multiplate scores with the agonists adenosine diphosphate, collagen, ristocetin and thrombin receptor activating peptide were obtained on 189 study days. The scores range was 0 to 126 . When the platelet count was below $33 \times 10^{9} / \mathrm{L}$ many samples had an area under the curve that was zero with at least one agonist. Platelet count, white blood cell count and reticulated platelet count were positively associated with the area under the curve. Interactions analysis for platelet count and reticulated platelet percent showed that the effect of reticulated platelets were dependent on platelet count.

Conclusion: We conclude that platelet count, white blood cells and reticulated platelets affected the results of the Multiplate analysis, and that for platelet counts below $33 \times 10^{9} / \mathrm{L}$ many Multiplate measurements were zero. Our results indicate that this analysis may not be applicable in routine evaluation of thrombocytopenic haemato-oncology patients.
\end{abstract}

\section{Introduction}

Multiplate is a platelet function analyser commonly used to diagnose platelet function disorders and to monitor antiplatelet treatment. Studies on healthy volunteers and patients with stable coronary artery disease (CAD) have demonstrated that platelet counts affect the Multiplate analysis [1-3]. Studies involving healthy volunteers and stable CAD patients on low dose aspirin treatment also report the impact of white blood cell counts (WBC) on Multiplate results $[2,4]$. Kander, et al. (2014) evaluated the effects of platelet transfusions on Multiplate results, and reported that the Multiplate area under the curve (AUC) was severely reduced in thrombocytopenic patients [5]. Some samples showed no aggregation response. Similar studies confirm these results $[5,6]$.

Patients with haematological malignancies develop thrombocytopenia as a consequence of the disease or treatment, and prophylactic platelet transfusions are often administered. Platelet count is used as trigger for platelet transfusions [7]. There are, however, uncertainties about the role of the platelet count as predictor of bleeding risk. Better indicators for predicting bleeding are thus required $[8,9]$.
We wanted to characterise the influence of haematological variables on Multiplate AUC in haemato-oncology patients with thrombocytopenia to evaluate if this test is a possible candidate to explore for an association with bleeding in this group of patients.

The primary objective of this pilot study was to characterise the effect of change in total platelet count (TPC) on Multiplate AUC. Secondary objectives were to characterise changes in Multiplate AUC related to variations in the haematological variables: white blood cell count (WBC), haemoglobin concentration $(\mathrm{Hb})$, mean platelet volume (MPV) and reticulated platelets.

Correspondence to: Tor Hervig, Department of Immunology and Transfusion Medicine, Haukeland University Hospital, Helse-Bergen HF, Norway, Tel: +47 559724 92; E-mail: tor.hervig@helse-bergen.no

Key words: haematological neoplasms, haematology tests, multiple electrode aggregometry, platelet function tests, thrombocytopenia

Received: March 13, 2017; Accepted: March 24, 2017; Published: March 27, 2017 


\section{Materials and methods}

This prospective, observational pilot study was approved by the local ethics committee. All subjects gave their written informed consent before participation. Adult patients (age $\geq 18$ ) with a haemato-oncological disease and thrombocytopenia with TPC $<50$ $\times 10^{9} / \mathrm{L}$, or expected to develop this grade of thrombocytopenia were approached. Patients with known congenital clotting disorders, regular use of anticoagulants in the study observation period, and immune thrombocytopenic purpura, were excluded.

Patients were enrolled consecutively from the Section for Haematology, Department of Medicine, at Haukeland University Hospital, Bergen, Norway from June 2013 until February 2014. For each participant, the study observation period lasted until platelet count recovery (unsupported platelet count $>50 \times 10^{9} / \mathrm{L}$ ), hospital discharge, or at most for 30 days of thrombocytopenia with TPC $<50 \times 10^{9} / \mathrm{L}$. Patients were eligible for inclusion in more than one chemotherapy cycle.

\section{Laboratory investigations}

Blood samples were collected from a central venous access (Hickman line) or an antecubital vein. Blood for Multiplate analysis was collected into $4 \mathrm{ml}$ Vacuette Sodium Heparin tubes (Greiner Bio-One $\mathrm{GmbH}$, Kremsmünster, Austria) every morning, Monday to Friday, in the study observation period. Sampling for haematology measurements was performed daily (including Saturdays and Sundays) into Vacuette 3 $\mathrm{ml}$ K2EDTA tubes (Greiner Bio-One $\mathrm{GmbH}$, Kremsmünster, Austria).

\section{Multiplate analysis}

Multiplate analysis is performed in a single-use test cell which incorporates two pairs of sensors, giving two parallel results and serving as a built-in quality assurance. The test cell is connected to the instrument with a sensor cable and the electrical resistance between the two sensor wires in a pair is recorded during the six-minute test period. The signal reaction in the Multiplate analyser is triggered by the adhesion of activated platelets to the surfaces of the sensor electrodes. The increase in electrical impedance caused by the attachment of platelets onto the Multiplate sensors is transformed to arbitrary aggregation units (AU) and plotted against time. Three variables are calculated: area under the curve (AUC), aggregation (AU), and velocity (AU/min). The most important variable is the AUC, which is recorded as Units or $\mathrm{U}$, an arbitrary unit $(10 \mathrm{AU} \times \mathrm{min}=1 \mathrm{U})$. AUC can have values from zero to well over 100. It is affected by the total height of the aggregation curve as well as by its slope and is best suited to express the overall platelet activity. The aggregation is the maximum height of the curve during the measurement period and the velocity is the maximum slope of the curve.

AUC was determined for four commercially available agonists that activate a range of receptors: adenosine diphosphate (ADP), collagen (COL), ristocetin (RISTO, high concentration, $0.77 \mathrm{mg} / \mathrm{ml}$ ) and thrombin receptor activating peptide (TRAP). ADP activates three different ADP-receptors [10] and COL activates collagen receptors, mainly integrin $\alpha 2 \beta 1$ and glycoprotein VI [11]. RISTO causes von Willebrand factor to bind to the glycoprotein Ib receptor [12], and TRAP stimulates the protease-activated-receptor-1 [13].

Multiplate analysis was performed according to the manufacturer's instructions and within 1 hour after collection. Reference values for Lithium Heparin are reported, as values for Sodium Heparin are not available from the manufacturer. Kaiser, et al. showed that Sodium
Heparin and Lithium Heparin anticoagulated samples give equal results for ADP in the first hour after sample collection [14]. During further storage Sodium Heparin conserved the results better than Lithium Heparin.

\section{Haematology analyses}

TPC, Hb, MPV and WBC were tested daily using a Cell-Dyn 4000 automated haematology analyser (Abbott Laboratories, Abbott Park, IL, US). Reticulated platelet percent (RPP) were analysed daily using a Cell-Dyn Sapphire automated haematology analyser (Abbott Laboratories, Abbott Park, IL, US). RPP and TPC were used to calculate reticulated platelet count (RPC).

\section{Statistical analyses}

This is a pilot study assessing previously unexplored associations between Multiplate analysis and haematological variables in thrombocytopenic haemato-oncology patients. Power calculations were not performed due to lack of data. Statistical analysis was performed with IBM SPSS statistics for Windows, version 23 (IBM Corp., Armonk, NY). Descriptive statistics are reported as quartiles $\left(Q_{1}, Q_{2}\right.$ and $\left.Q_{3}\right)$. Mixed model analysis was used to account for repeated measures and for patients being included twice. The AUC variables are strongly skewed to the right for all four agonists, with many measurements under the detection limit of the test $(\mathrm{AUC}=0)$ and a few values in the high end of the scale. To find the models that best fitted the criteria for linear mixed model analysis, the AUC variables (the outcome variables) were analysed without transformation, with ln transformation and with square root transformation against all relevant predictor variables. The analyses were repeated after $\ln$ transforming the predictor variables. From these analyses it was found that most models would benefit from an $\ln$ transformation of the AUC variables. As $\ln$ of zero is undefined, a constant, 1, was added to all AUC values to allow for statistical use of the results. The AUC variables are therefore $\ln$ of 1+AUC, but are referred to as AUC in the text.

WBC was the only predictor variable that was transformed in the final analyses, also by ln transformation, as this gave a better fitting model in most analyses. In the cases where WBC was beneath the detection limit of the analysis $\left(<0.2 \times 10^{9} / \mathrm{L}\right)$, the value was set to 0.1 to include it in the analysis. For the same reason TPC was set to 2 in the few cases where it was below the detection limit $\left(<5 \times 10^{9} / \mathrm{L}\right)$.

Analyses of interactions between RPP and TPC and between MPV and TPC were performed. To find the range of TPC for positive and negative effect of RPP and MPV on the outcome, the regression equation was derived with respect to RPP or MPV, respectively, and equated to zero. The solution of the equation gave the cut-point for TPC where the association between RPP or MPV and the outcome changed from negative to positive or the opposite.

\section{Results}

Ten patients ( 9 male, 1 female) were recruited. Four patients were included and gave written, informed consent in two subsequent chemotherapy cycles, giving 14 study observation periods (SOPs). Three patients ( 1 male, 2 female) declined to participate in the study.

The 10 individuals enrolled had a median age of 45.5 years (range 28-64). The diagnosis was acute myelogenous leukaemia (AML) in 4 individuals, myelodysplastic syndrome (MDS) in 2, MDS-AML in 2, multiple myeloma in 1 and histiocytic sarcoma in 1 . Of the patients who were included twice, two had AML and two had MDS. 
The treatment regimens for the 14 SOPs were remission induction chemotherapy in 11, consolidation chemotherapy in 1, allogeneic stem cell transplantation in 1 and autologous stem cell transplantation in 1.

In 10 of 14 inclusions the platelet count was $<50 \times 10^{9} / \mathrm{L}$ at the time of inclusion. The patients were followed throughout their 14 SOPs for a total of 298 days. The median observation period was 21 days, ranging from 7 to 39 days. There was a total of 261 study days with TPC $<50 \times 10^{9} / \mathrm{L}$. In 3 SOPs there were no days with TPC $>50 \times 10^{9} / \mathrm{L}$. Thrombocytopenia with TPC $<20 \times 10^{9} / \mathrm{L}$ was found on 119 study days and in all SOPs (median duration 8 days). TPC $<10 \times 10^{\%} / \mathrm{L}$ was found on 25 study days, occurring in 10 SOPs (median duration 1 day).

Platelet count was available for 294 (98.7\%) study days. 189 samples for Multiplate analyses were obtained from the participants during the study. The Multiplate laboratory results are summarised in table 1 .

Associations between haematology variables and Multiplate analysis

Summaries of the haematological laboratory results are shown in table 2 .

When the TPC fell below $33 \times 10^{9} / \mathrm{L}$, many samples had an AUC $=0$ with at least one agonist, while the 42 samples with TPC of 33 or above had detectable aggregation (AUC $>0$ ) with all four agonists. Table 3 presents the proportions of samples with $\mathrm{AUC}=0$ when $\mathrm{TPC}<33$ for each agonist. We found that all four $\ln$ transformed AUC variables were significantly correlated to TPC at the 0.01 level (2-tailed). Pearson's $r$ was around 0.7 and Spearman's $\rho$ around 0.8 for all four correlations (data not shown). The association between TPC and Multiplate AUC was positive for all agonists. The associations persisted after adjustment for $\ln$ of WBC, as presented in Table 4.

Ln of WBC shows a significant positive association with AUC for

Table 1. Summary of Multiplate results. Quartiles (Q) of Multiplate area under the curve (AUC) results in Units (U) for the four agonists adenosine diphosphate (ADP), collagen (COL), ristocetin (RISTO) and thrombin receptor activating peptide (TRAP).

\begin{tabular}{|l|c|c|c|c|c|}
\hline AUC agonist & $\mathbf{N}$ & $\mathbf{Q}_{\mathbf{1}}$ & $\mathbf{Q}_{\mathbf{2}}$ & $\mathbf{Q}_{\mathbf{3}}$ & Normal range (U) \\
\hline ADP & 189 & 0 & 2 & 6 & $55-117$ \\
\hline COL & 189 & 0 & 2 & 4 & $61-108$ \\
\hline RISTO & 185 & 0 & 1 & 5 & $65-116$ \\
\hline TRAP & 189 & 0 & 3 & 9 & $92-151$ \\
\hline
\end{tabular}

Table 2. Summary of haematology results. Quartiles $\left(\mathrm{Q}_{1}, \mathrm{Q}_{2}\right.$ and $\left.\mathrm{Q}_{3}\right)$ of the haematology variables platelet count (TPC), reticulated platelet percent (RPP), mean platelet volume (MPV), white blood cell count (WBC) and haemoglobin concentration (Hb). ${ }^{1}$ Not available: The laboratory has not established a normal range for reticulated platelets.

\begin{tabular}{|l|c|c|c|c|c|}
\hline Haematology variables & $\mathbf{N}$ & $\mathbf{Q}_{\mathbf{1}}$ & $\mathbf{Q}_{\mathbf{2}}$ & $\mathbf{Q}_{\mathbf{3}}$ & Normal range \\
\hline TPC $\left(\times 10^{9} / \mathrm{L}\right)$ & 294 & 15.00 & 23.00 & 31.25 & $145-387$ \\
\hline RPP (\%) & 176 & 3.12 & 4.57 & 6.71 & NA $^{1}$ \\
\hline MPV (fL) & 194 & 8.0 & 9.0 & 10.3 & $6.6-10.6$ \\
\hline WBC (x10 $/ \mathrm{L})$ & 294 & 0.1 & 0.3 & 0.5 & $3.5-11.0$ \\
\hline $\mathrm{Hb}(\mathrm{g} / \mathrm{dL})$ & 295 & 8.6 & 9.1 & 9.7 & $11.7-17.0$ \\
\hline
\end{tabular}

Table 3. Proportion of samples with a Multiplate area under the curve (AUC) $=0$ when the total platelet count (TPC) was less than $33 \times 10^{9} / \mathrm{L}$ and maximum TPC for which the AUC was 0 for each of the agonists adenosine diphosphate (ADP), collagen (COL), ristocetin (RISTO) and thrombin receptor activating peptide (TRAP). 'Proportion of samples with $\mathrm{AUC}=0$ when $\mathrm{TPC}<33 \times 109 / \mathrm{L} .{ }^{2}$ Maximum TPC for $\mathrm{AUC}=0$.

\begin{tabular}{|c|c|c|}
\hline Agonist & Proportion $^{\mathbf{1}}$ & TPC $^{\mathbf{2}}$ \\
\hline ADP & $37.7 \%$ & $27 \times 10^{9} / \mathrm{L}$ \\
\hline COL & $37.0 \%$ & $31 \times 10^{9} / \mathrm{L}$ \\
\hline RISTO & $55.6 \%$ & $32 \times 10^{9} / \mathrm{L}$ \\
\hline TRAP & $27.0 \%$ & $26 \times 10^{9} / \mathrm{L}$ \\
\hline
\end{tabular}

all agonists, also after adjusting for TPC (Table 4). The correlation coefficients between $\ln$ of WBC and $\ln$ of AUC for the four agonists were around 0.7-0.8 for Pearson's $r$ and around 0.5-0.6 for Spearman's $\rho$ (data not shown).

The results of the linear mixed model analyses for MPV, RPP, RPC and $\mathrm{Hb}$ non-adjusted, and adjusted for TPC and ln of WBC are shown in Table 5.

A significant negative association was found between MPV and AUC for all four agonists, and the association remained significant for ADP, COL and TRAP after adjusting for TPC and $\ln$ of WBC. Tests of interaction between TPC and MPV (Table 6) showed that the effect of MPV is different for different platelet counts. Taking this interaction into account, the association between MPV and AUC is positive when the TPC is above $20 \times 10^{9} / \mathrm{L}$ for ADP, 19 for COL, 17 for RISTO and 16 for TRAP, and negative when TPC is below these values. The association between TPC and AUC is positive for all measured values of MPV.

For $\mathrm{Hb}$ there was no significant association with AUC for any of the agonists, whether in unadjusted analysis or when adjusted for TPC and $\ln$ of WBC.

RPP had a significant negative association with AUC for RISTO, but not for the other agonists, persisting after adjusting for TPC and ln of WBC. Tests of interaction between TPC and RPP (Table 7) indicated that there is an interaction between the two variables for all four outcome variables. When we take this interaction into account, RPP was positively associated with AUC when TPC was above 12 $\times 10^{9} / \mathrm{L}$ for ADP, 14 for COL, 21 for RISTO and 11 for TRAP and negatively associated with AUC when TPC was below these values. TPC was positively associated with AUC for all values of RPP. When adjusting the interaction analysis for $\ln$ of WBC, TPC was no longer significant $(\mathrm{P}=0.085)$ for TRAP, but $\mathrm{RPP}$ and $\mathrm{RPC}$ remained significant for all agonists. The results for RPC could not be adjusted for TPC, since RPC is the product of TPC and RPP, but unadjusted and adjusted for $\ln$ of WBC there was a significant positive association for RPC with AUC for all four agonists. RPC was also positively associated with AUC for all agonists in the tests of interactions between TPC and RPP.

\section{Discussion}

The primary objective of the study was to characterise the effect of change in TPC on Multiplate AUC in thrombocytopenic haematooncology patients.

Previous studies of healthy volunteers and patients with stable CAD have found that TPC affects Multiplate results, especially when platelet counts are low, but no studies have been identified that tested the blood of patients with actual thrombocytopenia [1-4,15-17]. We found a significant association between TPC and AUC for all four agonists in patients with severe thrombocytopenia, which is in concordance with previous studies in healthy volunteers and CAD patients.

We found that many samples did not have detectable aggregation (AUC was 0) when the TPC was below $33 \times 10^{9} / \mathrm{L}$. Kander, et al. also found AUC $=0$ in some samples activated by ADP (AUC 0-9), but not by COL (AUC 1-16) or TRAP (AUC 1-18), in patients with bone marrow failure and platelet counts between 18 and 32 [5]. Stissing, et al. tested blood from healthy volunteers diluted with autologous plasma to platelet counts down to $25 \times 10^{9} / \mathrm{L}$ [1]. Exact AUC values are not reported, but a graph shows that for the samples with platelet count 25, AUC approaches 0 [1]. This seems to be in concordance with 
Table 4. Linear mixed model analysis of impact of total platelet count (TPC) and the natural logarithm (ln) of white blood cell count (WBC) on ln of $1+$ Multiplate area under the curve (AUC). Outcome variables are $\mathrm{ln}$ of 1+ Multiplate area under the curve (AUC) for the agonists adenosine diphosphate (ADP), collagen (COL), ristocetin (RISTO) and thrombin receptor activating peptide (TRAP). Outcome is listed as AUC, but is really $\ln (1+\mathrm{AUC})$. The results shown is the predicted change in outcome (B) when the predictor variable changes with 1 unit (and the other predictor variable is kept constant in adjusted analysis), the $95 \%$ confidence intervals of $\mathrm{B}$, and the probability (P) of finding B if the null hypothesis is true. Predictor variables are total platelet count (TPC) and the natural logarithm (ln) of the White Blood Cell Count (WBC).

\begin{tabular}{|c|c|c|c|c|c|c|c|c|c|}
\hline \multirow[b]{2}{*}{ Outcome } & \multirow[b]{2}{*}{ Predictor } & \multicolumn{4}{|c|}{ Unadjusted } & \multicolumn{4}{|c|}{ Mutually adjusted } \\
\hline & & $\mathrm{N}$ & B & $95 \% \mathrm{CI}$ & $\mathrm{P}$ & $\mathrm{N}$ & $\mathrm{B}$ & $95 \% \mathrm{CI}$ & $\mathrm{P}$ \\
\hline \multirow[t]{3}{*}{ AUC ADP } & - & - & - & - & - & 186 & - & - & - \\
\hline & TPC & 188 & 0.02 & $0.02,0.03$ & $<0.001$ & - & 0.01 & $0.01,0.02$ & $<0.001$ \\
\hline & Ln of WBC & 187 & 0.83 & $0.69,0.97$ & $<0.001$ & - & 0.59 & $0.46,0.72$ & $<0.001$ \\
\hline \multirow[t]{3}{*}{ AUC COL } & - & - & - & - & - & 186 & - & - & - \\
\hline & TPC & 188 & 0.02 & $0.02,0.03$ & $<0.001$ & - & 0.02 & $0.01,0.02$ & $<0.001$ \\
\hline & Ln of WBC & 187 & 0.60 & $0.46,0.74$ & $<0.001$ & - & 0.33 & $0.20,0.46$ & $<0.001$ \\
\hline \multirow[t]{3}{*}{ AUC RISTO } & - & - & - & - & - & 182 & - & - & - \\
\hline & TPC & 184 & 0.03 & $0.02,0.03$ & $<0.001$ & - & 0.02 & $0.02,0.02$ & $<0.001$ \\
\hline & Ln of WBC & 183 & 0.69 & $0.53,0.85$ & $<0.001$ & - & 0.38 & $0.24,0.52$ & $<0.001$ \\
\hline \multirow[t]{3}{*}{ AUC TRAP } & - & - & - & - & - & 186 & - & - & - \\
\hline & TPC & 188 & 0.02 & $0.02,0.03$ & $<0.001$ & - & 0.01 & $0.01,0.02$ & $<0.001$ \\
\hline & Ln of WBC & 187 & 0.96 & $0.80,1.11$ & $<0.001$ & - & 0.73 & $0.57,0.89$ & $<0.001$ \\
\hline
\end{tabular}

Table 5. Linear mixed model analysis of impact of haematologic variables on $\ln$ of $1+$ Multiplate area under the curve (AUC). Outcome variables are ln of $1+$ Multiplate area under the curve (AUC) for the agonists adenosine diphosphate (ADP), collagen (COL), ristocetin (RISTO) and thrombin receptor activating peptide (TRAP). The results shown is the predicted change in outcome (B) when the predictor variable changes with 1 unit (and the other predictor variables are kept constant in adjusted analysis), the $95 \%$ confidence interval of B, and the probability (P) of finding B if the null hypothesis is true. Predictor variables are mean platelet volume (MPV), reticulated platelet percent (RPP), reticulated platelet count (RPC) and haemoglobin concentration $(\mathrm{Hb})$ and in the adjusted analysis also total platelet count (TPC) and the natural logarithm (ln) of white blood cell count (WBC).

\begin{tabular}{|c|c|c|c|c|c|c|c|c|c|}
\hline \multirow[b]{2}{*}{ Outcome } & \multirow[b]{2}{*}{ Predictor } & \multicolumn{4}{|c|}{ Unadjusted } & \multicolumn{4}{|c|}{ Adjusted for TPC and In of WBC } \\
\hline & & $\mathbf{N}$ & B & $95 \% \mathrm{CI}$ & $\mathbf{P}$ & $\mathbf{N}$ & B & $95 \%$ CI & $\mathbf{P}$ \\
\hline \multirow[t]{4}{*}{ AUC ADP } & MPV & 167 & -0.20 & $-0.28,-0.12$ & $<0.001$ & 164 & -0.09 & $-0.15,-0.03$ & 0.012 \\
\hline & RPP & 151 & -0.05 & $-0.10,0.01$ & 0.078 & 148 & -0.01 & $-0.05,0.03$ & 0.725 \\
\hline & RPC & 150 & 0.01 & $0.01,0.01$ & $<0.001$ & - & - & - & - \\
\hline & $\mathrm{Hb}$ & 188 & 0.06 & $-0.09,0.20$ & 0.436 & 186 & 0.06 & $-0.05,0.17$ & 0.270 \\
\hline \multirow[t]{4}{*}{ AUC COL } & MPV & 168 & -0.18 & $-0.26,-0.11$ & $<0.001$ & 164 & -0.08 & $-0.14,-0.02$ & 0.008 \\
\hline & RPP & 151 & -0.04 & $-0.10,0.01$ & 0.088 & 148 & -0.01 & $-0.05,0.03$ & 0.595 \\
\hline & RPC & 150 & 0.01 & $0.01,0.01$ & $<0.001$ & - & - & - & - \\
\hline & $\mathrm{Hb}$ & 189 & 0.10 & $-0.04,0.25$ & 0.158 & 186 & 0.09 & $-0.02,0.21$ & 0.108 \\
\hline \multirow[t]{4}{*}{ AUC RISTO } & MPV & 165 & -0.19 & $-0.28,-0.10$ & $<0.001$ & 162 & -0.06 & $-0.13,0.01$ & 0.086 \\
\hline & RPP & 149 & -0.10 & $-0.16,-0.04$ & 0.001 & 146 & -0.06 & $-0.11-0.01$ & 0.017 \\
\hline & RPC & 148 & 0.01 & $0.00,0.01$ & $<0.001$ & - & - & - & - \\
\hline & $\mathrm{Hb}$ & 185 & -0.01 & $-0.18,0.15$ & 0.858 & 182 & 0.02 & $-0.11,0.15$ & 0.715 \\
\hline \multirow[t]{4}{*}{ AUC TRAP } & MPV & 167 & -0.18 & $-0.28,-0.09$ & $<0.001$ & 164 & -0.09 & $-0.16,-0.02$ & 0.012 \\
\hline & RPP & 151 & -0.04 & $-0.10,0.02$ & 0.245 & 148 & 0.00 & $-0.05,0.05$ & 0.990 \\
\hline & $\mathrm{RPC}$ & 150 & 0.01 & $0.01,0.01$ & $<0.001$ & - & - & - & - \\
\hline & $\mathrm{Hb}$ & 189 & 0.14 & $-0.02,0.30$ & 0.083 & 186 & 0.10 & $-0.02,0.22$ & 0.106 \\
\hline
\end{tabular}

our findings.

Laine, et al. did a study on patients with Hantavirus infection who had platelet counts varying from normal to severe thrombocytopenia with TPC down to 17 in the study samples [6]. They found detectable aggregation (AUC $>0$ ) in all samples, with AUC $=5$ for RISTO as the lowest measurement. For ADP, COL and TRAP the lowest measurements were 21 (ADP, COL) and 18 (TRAP) [6]. These responses seem to be a little higher than what we found, which may be due to different study populations.

Secondary objectives of the study were to characterise changes in Multiplate AUC related to variations in the haematologic variables white blood cell count (WBC), mean platelet volume (MPV), reticulated platelets and haemoglobin concentration $(\mathrm{Hb})$.

WBC was found to have a highly significant association with AUC for all agonists. Würtz, et al. [2] and Rubak, et al. [4] also found a significant effect of WBC on AUC. Seyfert, et al [15] did not find a significant effect of WBC on AUC in a study on healthy volunteers.
The impact of white blood cells on platelet aggregation is controversial. White blood cells have the capacity to release substances that can both enhance (e.g., cathepsin G, reactive oxygen species) and inhibit platelet function (e.g., nitric oxide), and both effects have been reported in vitro [18-21]. Our findings support the hypothesis that leucocytes have an enhancing effect on platelet aggregation.

According to Faraday, et al. this effect can be explained by the interaction of platelet P-selectin with leukocyte P-selectin glycoprotein ligand-1 leading to an enhanced platelet agonist-induced aggregation and thromboxane release [22].

Adjustment for TPC and ln of WBC was used in the analysis of all the remaining predictor variables. The reason for the TPC adjustment was the known effects of this variable, and the WBC adjustment was done because we found that the white blood cell count has a highly significant effect on the outcome of the Multiplate analysis in this patient group.

For MPV a significant negative association with AUC was found 
Table 6. Linear mixed model analysis to test for interactions between total platelet count (TPC) and mean platelet volume (MPV) with and without adjustment for the natural logarithm (ln) of white blood cells (WBC). Outcome variables are ln of 1+ Multiplate area under the curve (AUC) for the agonists adenosine diphosphate (ADP), collagen (COL), ristocetin (RISTO) and thrombin receptor activating peptide (TRAP). The results shown is the predicted change in outcome (B) when the predictor variable changes with 1 unit (and the other predictor variables are kept constant), the $95 \%$ confidence interval of $\mathrm{B}$, and the probability $(\mathrm{P})$ of finding $\mathrm{B}$ if the null hypothesis is true.

\begin{tabular}{|c|c|c|c|c|c|c|c|c|c|}
\hline \multirow[b]{2}{*}{ Outcome } & \multirow[b]{2}{*}{ Predictor } & \multicolumn{4}{|c|}{ Mutual adjustment } & \multicolumn{4}{|c|}{ Also adjusted for In of WBC } \\
\hline & & $\mathbf{N}$ & B & $95 \% \mathrm{CI}$ & $\mathbf{P}$ & $\mathbf{N}$ & B & $95 \% \mathrm{CI}$ & $\mathbf{P}$ \\
\hline \multirow[t]{4}{*}{ AUC ADP } & - & 166 & - & - & - & 164 & - & - & - \\
\hline & TPC & - & -0.03 & $-0.06,-0.01$ & 0.020 & - & -0.03 & $-0.05,-0.01$ & 0.002 \\
\hline & MPV & - & -0.14 & $-0.21,-0.07$ & $<0.001$ & - & -0.15 & $-0.21,-0.09$ & $<0.001$ \\
\hline & TPC x MPV & - & 0.01 & $0.00,0.01$ & $<0.001$ & - & 0.01 & $0.00,0.01$ & $<0.001$ \\
\hline \multirow[t]{4}{*}{ AUC COL } & - & 166 & - & - & - & 164 & - & - & - \\
\hline & TPC & - & -0.04 & $-0.06,-0.02$ & 0.001 & - & -0.04 & $-0.06,-0.02$ & 0.001 \\
\hline & MPV & - & -0.15 & $-0.22,-0.09$ & $<0.001$ & - & -0.15 & $-0.21,-0.09$ & $<0.001$ \\
\hline & TPC x MPV & - & 0.01 & $0.01,0.01$ & $<0.001$ & - & 0.01 & $0.00,0.01$ & $<0.001$ \\
\hline \multirow[t]{4}{*}{ AUC RISTO } & - & 164 & - & - & - & 162 & - & - & - \\
\hline & TPC & - & -0.04 & $-0.07,-0.02$ & 0.001 & - & -0.04 & $-0.06,-0.01$ & 0.002 \\
\hline & MPV & - & -0.15 & $-0.22,-0.07$ & $<0.001$ & - & -0.14 & $-0.21,-0.07$ & $<0.001$ \\
\hline & TPC x MPV & - & 0.01 & $0.01,0.01$ & $<0.001$ & - & 0.01 & $0.00,0.01$ & $<0.001$ \\
\hline \multirow[t]{4}{*}{ AUC TRAP } & - & 166 & - & - & - & 164 & - & - & - \\
\hline & TPC & - & -0.04 & $-0.07,-0.01$ & 0.018 & - & -0.04 & $-0.07,-0.02$ & 0.001 \\
\hline & MPV & - & -0.13 & $-0.22,-0.05$ & 0.002 & - & -0.16 & $-0.23,-0.09$ & $<0.001$ \\
\hline & TPC x MPV & - & 0.01 & $0.00,0.01$ & $<0.001$ & - & 0.01 & $0.00,0.01$ & $<0.001$ \\
\hline
\end{tabular}

Table 7. Linear mixed model analysis to test for interactions between total platelet count (TPC) and reticulated platelet percent (RPP) with and without adjustment for the natural logarithm (ln) of white blood cells (WBC). Outcome variables are ln of 1+ Multiplate area under the curve (AUC) for the agonists adenosine diphosphate (ADP), collagen (COL), ristocetin (RISTO) and thrombin receptor activating peptide (TRAP). The results shown is the predicted change in outcome (B) when the predictor variable changes with 1 unit (and the other predictor variables are kept constant), the $95 \%$ confidence interval of $\mathrm{B}$, and the probability $(\mathrm{P})$ of finding $\mathrm{B}$ if the null hypothesis is true.

\begin{tabular}{|c|c|c|c|c|c|c|c|c|c|}
\hline \multirow[b]{2}{*}{ Outcome } & \multirow[b]{2}{*}{ Predictor } & \multicolumn{4}{|c|}{ Mutual adjustment } & \multicolumn{4}{|c|}{ Also adjusted for In of WBC } \\
\hline & & $\mathbf{N}$ & B & $95 \%$ CI & $\mathbf{P}$ & $\mathbf{N}$ & B & $95 \%$ CI & $\mathbf{P}$ \\
\hline \multirow[t]{4}{*}{ AUC ADP } & - & 150 & - & - & - & 148 & - & - & - \\
\hline & TPC & - & 0.01 & $0.01,0.02$ & $<0.001$ & - & 0.01 & $0.00,0.01$ & 0.004 \\
\hline & RPP & - & -0.10 & $-0.15,-0.05$ & $<0.001$ & - & -0.10 & $-0.15,-0.06$ & $<0.001$ \\
\hline & TPC x RPP & - & 0.01 & $0.01,0.01$ & $<0.001$ & - & 0.01 & $0.01,0.01$ & $<0.001$ \\
\hline \multirow[t]{4}{*}{ AUC COL } & - & 150 & - & - & - & 148 & - & - & - \\
\hline & TPC & - & 0.01 & $0.01,0.02$ & $<0.001$ & - & 0.01 & $0.00,0.01$ & 0.001 \\
\hline & RPP & - & -0.09 & $-0.14,-0.04$ & 0.001 & - & -0.09 & $-0.14,-0.04$ & 0.001 \\
\hline & TPC x RPP & - & 0.01 & $0.00,0.01$ & $<0.001$ & - & 0.01 & $0.00,0.01$ & $<0.001$ \\
\hline \multirow[t]{4}{*}{ AUC RISTO } & - & 148 & - & - & - & 146 & - & - & - \\
\hline & TPC & - & 0.01 & $0.01,0.02$ & $<0.001$ & - & 0.01 & $0.00,0.01$ & $<0.001$ \\
\hline & RPP & - & -0.13 & $-0.19,-0.07$ & $<0.001$ & - & -0.14 & $-0.19,-0.08$ & $<0.001$ \\
\hline & TPC x RPP & - & 0.01 & $0.00,0.01$ & $<0.001$ & - & 0.01 & $0.00,0.01$ & $<0.001$ \\
\hline \multirow[t]{4}{*}{ AUC TRAP } & - & 150 & - & - & - & 148 & - & - & - \\
\hline & TPC & - & 0.01 & $0.00,0.02$ & 0.001 & - & 0.00 & $-0.00,0.01$ & 0.085 \\
\hline & RPP & - & -0.10 & $-0.16,-0.04$ & 0.002 & - & -0.10 & $-0.15,-0.04$ & $<0.001$ \\
\hline & TPC x RPP & - & 0.01 & $0.01,0.01$ & $<0.001$ & - & 0.01 & $0.01,0.01$ & $<0.001$ \\
\hline
\end{tabular}

with ADP, COL and TRAP after adjusting for TPC and ln of WBC. This is the opposite of what Grove, et al. [17] found for ADP and COL in patients with stable CAD. The patients in the study by Grove, et al. had normal platelet counts, while the patients in this study have a severe thrombocytopenia on the majority of study days. This may be the reason for the contradicting results. When taking into account the interaction between MPV and TPC, the association between MPV and AUC is positive when TPC is above $16-20 \times 10^{9} / \mathrm{L}$ for the four agonists and negative when TPC is below these values. This finding may support the theory that the low platelet counts in the patients in this study are the reason for the negative association of MPV with AUC when the interaction between MPV and TPC is not taken into account.

To account for interactions between TPC and RPP, we analysed an interaction model with these two variables and their product, RPC. The three variables were all significantly associated with AUC for all four agonists, indicating that there is an interaction between TPC and RPP, and that the effect of TPC is different for different values of RPP. When taking the interaction into account, there is a significant association between RPP and AUC for all four agonists, and the association is positive when TPC is above a certain value $\left(11-21 \times 10^{9}\right.$ /L for the different agonists). We are not aware of any other studies where interaction models have been analysed. When the interaction analysis was adjusted for $\ln$ of WBC, TPC no longer had a significant association with AUC for TRAP, but all other associations remained highly significant. This may indicate that for strong agonists, reticulated platelet count can be of greater importance than total platelet count in predicting aggregation response.

RPC was significantly positively associated with AUC whether unadjusted or adjusted, and also in the interaction analysis. This corresponds with the results reported from Grove, et al. [17] in a study 
on patients with stable CAD. Grove, et al. did not adjust for other variables. Estcourt, et al. found that haemato-oncology patients with higher counts of absolute immature platelets had less bleeding than patients with lower counts [9], which support our finding.

$\mathrm{Hb}$ did not have a significant association with AUC for any of the four agonists, which is in concordance with the findings of Seyfert, et al. [15], who tested healthy volunteers with arachidonic acid, ADP and collagen as agonists.

The main limitation of our study, but also our main finding, is that a high proportion of the Multiplate analyses had an aggregation of 0 . However, a high total number of study days with Multiplate analysis were undertaken. In our pilot study, only 10 patients were included, all being treated for haematological malignancies. However, we acknowledge heterogeneity in diagnosis and treatment regimens in our patients.

This study shows that Multiplate aggregometry in thrombocytopenic haemato-oncologic patients is dependent not only on platelet count, but also on WBC. Reticulated platelets also affect the results of Multiplate analysis, and we showed that there was an interaction between TPC and RPP. Many Multiplate measures were zero, which indicate that this method might not be applicable in this patient group unless the sensitivity can be enhanced. Our results provide a baseline for further evaluation. Larger clinical studies will be needed to address the role of point-of-care analysis in assessment of bleeding risk in thrombocytopenic patients with haematological malignancies.

\section{Acknowledgements}

This work was supported by a grant from the University of Bergen.

We thank the Laboratory for Clinical Biochemistry at Haukeland University Hospital for the analysis of blood samples and the nurses at the Haematology ward at Haukeland University Hospital for their support and sample collection.

ENO contributed to the design of the study, the draft of the protocol, data collection, sample analysis, statistical analysis of the results and preparation of the manuscript.

TOA contributed to the design of the study, the draft of the protocol and the preparation of the manuscript. SJS contributed to the design of the study and the preparation of the manuscript. GEE contributed to the design of the study, the statistical analysis of the results and to the preparation of the manuscript. TH contributed to the design of the study, the draft of the protocol and to the preparation of the manuscript.

\section{References}

1. Stissing T, Dridi NP, Ostrowski SR, Bochsen L, Johansson PI (2011) The influence of low platelet count on whole blood aggregometry assessed by Multiplate. Clin Appl Thromb Hemost 17: E211-217.[Crossref]

2. Würtz M, Hvas AM, Kristensen SD, Grove EL (2012) Platelet aggregation is dependent on platelet count in patients with coronary artery disease. Thromb Res 129: 56-61. [Crossref]

3. Femia EA, Scavone M, Lecchi A, Cattaneo M(2013) Effect of platelet count on platelet aggregation measured with impedance aggregometry (Multiplate analyzer) and with light transmission aggregometry. $J$ Thromb Haemost 11:2193-2196.

4. Rubak P, Villadsen K, Hvas AM (2012) Reference intervals for platelet aggregation assessed by multiple electrode platelet aggregometry. Thromb Res 130: 420-423. [Crossref]

5. Kander T, Tanaka KA, Norström E, Persson J, Schött U (2014) The effect and duration of prophylactic platelet transfusions before insertion of a central venous catheter in patients with bone marrow failure evaluated with point-of-care methods and flow cytometry. Anesth Analg 119: 882-890.[Crossref]

6. Laine O, Joutsi-Korhonen L, Lassila R, Koski T, Huhtala H, et al. (2015) Hantavirus infection-induced thrombocytopenia triggers increased production but associates with impaired aggregation of platelets except for collagen. Thromb Res 136: 1126-1132. [Crossref]

7. Kreuger AL, Middelburg RA, Zwaginga JJ (2015) Clinical practice of platelet transfusions in haemato-oncology. Vox Sang 109: 91-94.[Crossref]

8. Friedmann AM, Sengul H, Lehmann H, Schwartz C, Goodman S (2002) Do basic laboratory tests or clinical observations predict bleeding in thrombocytopenic oncology patients? A reevaluation of prophylactic platelet transfusions. Transfus Med Rev 16: 34-45.[Crossref]

9. Estcourt LJ, Stanworth SJ, Harrison P, Powter G, McClure M, et al. (2014) Prospective observational cohort study of the association between thromboelastometry, coagulation and platelet parameters and bleeding in patients with haematological malignancies- the ATHENA study. Br J Haematol 166: 581-591.[Crossref]

10. Murugappa S, Kunapuli SP (2006) The role of ADP receptors in platelet function. Front Biosci 11: 1977-1986.[Crossref]

11. Clemetson KJ, Clemetson JM (2001) Platelet collagen receptors. Thromb Haemost 86 189-197.[Crossref]

12. Kroll MH, Harris TS, Moake JL, Handin RI, Schafer AI (1991) von Willebrand factor binding to platelet GpIb initiates signals for platelet activation. J Clin Invest 88: 1568 1573.[Crossref]

13. Vu TK, Hung DT, Wheaton VI, Coughlin SR (1991) Molecular cloning of a functional thrombin receptor reveals a novel proteolytic mechanism of receptor activation. Cell64: 1057-1068.[Crossref]

14. Kaiser AF, Neubauer H, Franken CC, Krüger JC, Mügge A, et al. (2012) Which is the best anticoagulant for whole blood aggregometry platelet function testing? Comparison of six anticoagulants and diverse storage conditions. Platelets23: 359-367.[Crossref]

15. Seyfert UT, Haubelt H, Vogt A, Hellstern P (2007) Variables influencing Multiplate(TM) whole blood impedance platelet aggregometry and turbidimetric platelet aggregation in healthy individuals. Platelets 18: 199-206.[Crossref]

16. Hanke AA, Roberg K, Monaca E, Sellmann T, Weber CF, et al. (2010) Impact of platelet count on results obtained from multiple electrode platelet aggregometry (Multiplate). Eur J Med Res 15: 214-219.[Crossref]

17. Grove EL, Hvas AM, Mortensen SB, Larsen SB, Kristensen SD (2011) Effect of platelet turnover on whole blood platelet aggregation in patients with coronary artery disease. J Thromb Haemost 9: 185-191.[Crossref]

18. Selak MA, Chignard M, Smith JB (1988) Cathepsin G is a strong platelet agonist released by neutrophils. Biochem J251: 293-299. [Crossref]

19. Salvemini D, de Nucci G, Gryglewski RJ, Vane JR (1989) Human neutrophils and mononuclear cells inhibit platelet aggregation by releasing a nitric oxide-like factor Proc Natl Acad Sci U S A 86: 6328-6332.[Crossref]

20. Pratico D, Iuliano L, Alessandri C, Camastra C, Violi F(1993) Polymorphonuclear leukocyte-derived $\mathrm{O} 2$-reactive species activate primed platelets in human whole blood. Am. J Physiol264:H1582-H15827.

21. Valles J, Santos MT, Marcus AJ, Safier LB, Broekman MJ, et al. (1993) Downregulation of human platelet reactivity by neutrophils. Participation of lipoxygenase derivatives and adhesive proteins. $J$ Clin Invest 92: 1357-1365.[Crossref]

22. Faraday N, Scharpf RB, Dodd-o JM, Martinez EA, Rosenfeld BA, et al. (2001) Leukocytes can enhance platelet-mediated aggregation and thromboxane release via interaction of P-selectin glycoprotein ligand 1 with P-selectin. Anesthesiology94: 145151.[Crossref]

Copyright: (C)2017 Opheim EN. This is an open-access article distributed under the terms of the Creative Commons Attribution License, which permits unrestricted use, distribution, and reproduction in any medium, provided the original author and source are credited. 